Proceedings of the Edinburgh Mathematical Society (2002) 45, 397-409 (C)

DOI:10.1017/S0013091501000189 Printed in the United Kingdom

\title{
INFINITELY MANY SOLUTIONS TO THE NEUMANN PROBLEM FOR ELLIPTIC EQUATIONS INVOLVING THE $p$-LAPLACIAN AND WITH DISCONTINUOUS NONLINEARITIES
}

\author{
PASQUALE CANDITO \\ Dipartimento di Matematica, Università di Messina, Contrada Papardo, \\ Salita Sperone, 98166 Sant' Agata (ME), Italy and \\ Dipartimento di Patrimonio Architettonico e Urbanistico Facoltà di Architettura, \\ Università di Reggio Calabria, Salita Melissari (Feo di Vito), \\ 89124 Reggio Calabria, Italy (candito@mailer.ing.unirc.it)
}

(Received 12 February 2001)

\begin{abstract}
In this paper, we establish the existence of infinitely many solutions to a Neumann problem involving the $p$-Laplacian and with discontinuous nonlinearities. The technical approach is mainly based on a very recent result on critical points for possibly non-smooth functionals in a Banach space due to Marano and Motreanu, namely Theorem 1.1 in a paper that is to appear in the journal J. Diff. Eqns (see Theorem 2.3 in the body of this paper). Some applications are presented.

Keywords: critical points of non-smooth functions; p-Laplacian; elliptic problems with discontinuous nonlinearities

AMS 2000 Mathematics subject classification: Primary 35A15; 35J65; 35R05
\end{abstract}

\section{Introduction}

Throughout the sequel, $\Omega$ is a non-empty bounded open set of the real Euclidean space $\mathbb{R}^{n}, n \geqslant 3$, with boundary of class $C^{\infty}$, a belongs to $L^{\infty}(\Omega)$, with $\operatorname{ess} \inf \Omega a>0 ; \alpha(x)$, $\beta(x)$ lie in $L^{1}(\Omega)$ with $\min \{\alpha(x), \beta(x)\} \geqslant 0$ a.e. in $\Omega$, and $\left.p \in\right] n,+\infty[$. Consider the following problem

$$
\left.\begin{array}{c}
\Delta_{p} u-a(x)|u|^{p-2} u=\alpha(x) f(u)+\beta(x) g(u) \text { in } \Omega, \\
\frac{\partial u}{\partial v}=0 \quad \text { on } \partial \Omega,
\end{array}\right\}
$$

where $\Delta_{p} u=\operatorname{div}\left(|\nabla u|^{p-2} \nabla u\right)$ is the $p$-Laplacian, $\nu$ is the outer unit normal to $\partial \Omega$ and $f, g: \mathbb{R} \rightarrow \mathbb{R}$ are two locally essentially bounded functions. Denote by $D_{f}$ (respectively, $D_{g}$ ) the set of all discontinuity points of $f$ (respectively, $g$ ). 
Let us recall that a weak solution of problem $\left(N_{p}\right)$ is any $u \in W^{1, p}(\Omega)$ such that

$$
\begin{aligned}
&-\int_{\Omega}\left(|\nabla u(x)|^{p-2} \nabla u(x)\right.\nabla v(x)) \mathrm{d} x-\int_{\Omega}\left(a(x)|u(x)|^{p-2} u(x) v(x)\right) \mathrm{d} x \\
&=\int_{\Omega}(\alpha(x) f(u(x))+\beta(x) g(u(x))) v(x) \mathrm{d} x, \quad \forall v \in W^{1, p}(\Omega) .
\end{aligned}
$$

To the best of our knowledge, no investigation has been devoted to establishing the existence of infinitely many solutions to such a problem. Actually, we can only mention the papers $[\mathbf{1 0}, \mathbf{1 3}]$ (see also $[\mathbf{1}]$ ). Both of them give a positive response to the question whenever $f$ and $g$ are two continuous functions. Precisely, in [13] the goal is achieved by a direct application of a general result on critical points due to Ricceri, see Theorem 2.5 of [12]; whereas in [10] the situation is rather different. There, the functions $f$ and $g$ treated belong to $L_{\text {loc }}^{\infty}(\mathbb{R})$. Moreover, Marano and Motreanu extend the above-mentioned critical-points result to a family of functionals in a Banach space which are possibly nonsmooth (see Theorem 1.1 of [10] or Theorem 2.3 below, see also [11]). In this connection, thanks to this latter result, the conclusion has been obtained by noting that problem $\left(N_{p}\right)$ represents the special case of a variational-hemivariational inequality involving $f$ and $g$, provided that $f$ and $g$ belong to $C^{\circ}(\mathbb{R})$.

The aim of the present paper is to prove Theorems 1 and 2 of $[\mathbf{1 3}]$ assuming, instead of the continuity of $f$ and $g$, a more general condition which is compatible with the fact that the sets $D_{f}$ and $D_{g}$ can be uncountable.

Exploiting the non-smooth framework developed in [10], the proof of our main results will be performed in three steps. First, reasoning as in the proof of Theorem 2.1 of [10], and using Theorem 2.3, we show that an appropriate locally Lipschitz functional $J$ admits a sequence of critical points (in the Chang sense, see [4]). Next, using concepts and results from the critical-point theory for non-smooth locally Lipschitz functionals developed by Clarke and Chang, see Proposition 2.1 below, we adapt the technique introduced to prove Theorem 4.2 of $[\mathbf{9}]$ in solving a suitable multivalued version of problem $\left(N_{p}\right)$ (see also $[\mathbf{2}, \mathbf{7}]$ regarding the Dirichlet problem). Subsequently, under additional assumptions in comparison with Theorems 2.1 and 2.2 of [10], we show that each critical point of the functional $J$ turns out to be a weak solution of the above problem. Finally, to the end of emphasizing the usefulness of our results in solving several concrete cases, we will present three examples with $g=0$. The first of them deals with a function having an uncountable bounded set of discontinuities of the first kind. The second one treats the case of just one point of discontinuity of second kind. A function having a countable and unbounded set of discontinuities of first kind is investigated in the last example.

The paper is organized in four sections. Notation, basic definitions and preliminary results are collected in $\S 2$. The main results are presented in $\S 3$. The last section is devoted to explaining the examples.

\section{Basic definitions and preliminary results}

Let $(X,\|\cdot\|)$ be a real Banach space. We denote by $X^{*}$ the dual space of $X$, while $\langle\cdot, \cdot\rangle$ stands for the duality pairing between $X^{*}$ and $X$. A function $J: X \rightarrow \mathbb{R}$ is called locally 
Lipschitz when to every $u \in X$ there corresponds a neighbourhood $U$ of $u$ and a constant $L_{U} \geqslant 0$ such that

$$
|J(u)-J(v)| \leqslant L_{U}\|u-v\| \quad \forall u, v \in U .
$$

Write, if $\xi \in \mathbb{R}$,

$$
F(\xi):=\int_{0}^{\xi} f(t) \mathrm{d} t, \quad G(\xi):=\int_{0}^{\xi} g(t) \mathrm{d} t .
$$

It is easy to prove that $F$ and $G$ are locally Lipschitz whenever $f$ and $g$ are two locally essentially bounded functions. If $u, v \in X$, the symbol $J^{\circ}(u ; v)$ indicates the generalized directional derivative of $J$ at the point $u$ along the direction $v$, namely

$$
J^{\circ}(u ; v)=\limsup _{w \rightarrow u, t \rightarrow 0^{+}} \frac{J(w+t v)-J(v)}{t} .
$$

The generalized gradient of the functional $J$ at $u$, denoted by $\partial J(u)$, is the set

$$
\partial J(u):=\left\{u^{*} \in X^{*}:\left\langle u^{*}, v\right\rangle \leqslant J^{\circ}(u ; v) \forall v \in X\right\} .
$$

We say that $u \in X$ is a critical point of $J$ if it fulfils the inequality

$$
J^{\circ}(u ; v) \geqslant 0 \quad \forall v \in X .
$$

It is well known that if $J$ is continuously Gâteaux differentiable at $u$, then $\partial J(u)=J^{\prime}(u)$, $J^{\circ}(u ; v)$ coincides with the directional derivative $J^{\prime}(u ; v)$ for each $v \in X$, and (2.1) becomes $J^{\prime}(u)=0$. Furthermore, it is easy to prove that each local minimum is also a critical point of $J$ (see Proposition 2.1 of [9]). In this framework the following proposition collects some basic properties about the directional generalized derivative.

Proposition 2.1. Let $I, J: X \rightarrow \mathbb{R}$ be two locally Lipschitz functions. The following assertions hold.

$\left(p_{1}\right)(I+J)^{\circ}(u ; v) \leqslant I^{\circ}(u ; v)+J^{\circ}(u ; v) \forall v \in X$.

$\left(p_{2}\right)$ If $I$ is continuously Gâteaux differentiable at $u$, then

$$
(I+J)^{\circ}(u ; v)=I^{\prime}(u ; v)+J^{\circ}(u ; v) \quad \forall v \in X .
$$

For a thorough treatment of this topic we refer to $[\mathbf{4 , 5}]$ and the references cited therein. On the space $W^{1, p}(\Omega)$ we consider the norm

$$
\|u\|:=\left(\int_{\Omega}\left(|\nabla u(x)|^{p} \mathrm{~d} x+a(x)|u(x)|^{p}\right) \mathrm{d} x\right)^{1 / p}
$$

which is clearly equivalent to the usual one, while we equip the space $C^{0}(\bar{\Omega})$ with the norm $\|u\|_{C^{0}(\bar{\Omega})}:=\sup _{u \in \bar{\Omega}}|u(x)|$. Since $p>n$, we have

$$
c:=\sup _{u \in W^{1, p}(\Omega) \backslash\{0\}} \frac{\|u\|_{C^{0}(\bar{\Omega})}}{\|u\|}<+\infty .
$$


Moreover, if $\Omega$ is a convex set whose diameter is less than or equal to

$$
n^{1 / p}\left(\frac{p-n}{p-1}\right)^{1-(1 / p)}
$$

then the following upper bounded estimate holds

$$
c \leqslant\left(\frac{2^{p-1}}{\min \left\{1, \operatorname{essinf}_{\Omega} a\right\} m(\Omega)}\right)^{1 / p} .
$$

For more details on the constant $c$ see [3]. Let $E$ be a subset of $\mathbb{R}^{n}$, from now on, 'measurable' always means Lebesgue measurable and $m(E)$ stands for the measure of $E$. As usual, a function $l: \Omega \times \mathbb{R} \rightarrow \mathbb{R}$ is said to be 'superpositionally measurable' if, for every measurable function $u: \Omega \rightarrow \mathbb{R}$, the function $l(x, u(x))$ is measurable. If for almost every $x$ in $\Omega, l(x, \cdot)$ is locally essentially bounded, write

$$
l^{-}(x, t):=\lim _{\delta \rightarrow 0^{+}|t-z|<\delta} \operatorname{ess} l(x, z), \quad l^{+}(x, t):=\lim _{\delta \rightarrow 0^{+}|t-z|<\delta} \operatorname{esup}(x, z)
$$

for each $t \in \mathbb{R}$. It is a simpler matter to see that $l^{-}(x, \cdot)$ and $l^{+}(x, \cdot)$ are, respectively, lower semicontinuous and upper semicontinuous.

Proposition 2.2. Let $u \in W^{1, p}(\Omega)$ and let $E$ be a measurable subset of $\mathbb{R}$ such that $m(E)=0$. Then $\Delta_{p} u=0$ at almost all $x \in u^{-1}(E)$.

Proof. It is well known that there exists a set $H$ of type $G_{\delta}$ such that $E \subset H$ and $m(H)=0$. Thus, owing to Lemma 1 of $[6]$, one has $\nabla u(x)=0$ a.e. on $u^{-1}(H)$. From this the conclusion follows at once (see also $[\mathbf{2}]$ and the references cited therein).

Now, for the reader's convenience, we state a non-smooth version due to Marano and Motreanu of a very recent result on critical points of Ricceri, which represents the main tool to investigate problem $\left(N_{p}\right)$.

Let $\tilde{X}$ be a real Banach space such that $X$ is compactly embedded in $\tilde{X}$, let $\Phi: \tilde{X} \rightarrow \mathbb{R}$ and $\Psi: X \rightarrow \mathbb{R}$ be two locally Lipschitz functions. Write, provided $\rho>\inf _{X} \Psi$,

$$
\varphi(\rho):=\inf _{u \in \Psi^{-1}(]-\infty, \rho[)} \frac{\Phi(u)-\inf _{\frac{\left(\Psi^{-1}(]-\infty, \rho[)\right)_{\mathrm{w}}}{\rho}} \Phi(v)}{\rho-\Psi(u)},
$$

where $\overline{\left(\Psi^{-1}(]-\infty, \rho[)\right)_{\mathrm{w}}}$ stands for the weak closure of $\Psi^{-1}(]-\infty, \rho[)$,

$$
\gamma:=\liminf _{\rho \rightarrow+\infty} \varphi(\rho), \quad \delta:=\liminf \rho \rightarrow\left(\inf _{X} \Psi\right)^{+} \varphi(\rho) .
$$

Theorem 2.3 (Theorem 1.1 of [10]). Let $X$ be reflexive and let $\Psi$ be weakly sequentially lower semicontinuous and coercive. Then the following assertions hold.

(a) For every $\rho>\inf _{X} \Psi$ and every $\lambda>\varphi(\rho)$ the function $\Phi+\lambda \Psi$ has a critical point (local minima) lying in $\Psi^{-1}(]-\infty, \rho[)$. 
(b) If $\gamma<+\infty$, then, for each $\lambda>\gamma$, either

$\left(\mathrm{b}_{1}\right) \Phi+\lambda \Psi$ possesses a global minimum, or

$\left(\mathrm{b}_{2}\right)$ there is a sequence $\left\{u_{k}\right\}$ of critical points (local minima) such that

$$
\lim _{k \rightarrow+\infty} \Psi\left(u_{k}\right)=+\infty
$$

(c) If $\delta<+\infty$, then, for each $\lambda>\delta$, either

$\left(c_{1}\right) \Phi+\lambda \Psi$ has a local minimum, which is also a global minimum of $\Psi$, or

$\left(c_{2}\right)$ there is a sequence $\left\{u_{k}\right\}$ of pairwise distinct critical points (local minima) of $\Phi+\lambda \Psi$, with $\lim _{k \rightarrow+\infty} \Psi\left(u_{k}\right)=\inf _{X} \Psi$, which weakly converges to a global minimum of $\Psi$.

\section{Main results}

We now establish the main results.

Theorem 3.1. Let $f, g: \mathbb{R} \rightarrow \mathbb{R}$ be two locally essentially bounded functions, which are possibly discontinuous in a set having Lebesgue measure zero, namely $D_{f}$ and $D_{g}$, respectively. Assume that the following assertions hold.

$\left(\mathrm{d}_{1}\right) \inf _{\xi \in \mathbb{R}} G(\xi) \geqslant 0$.

$\left(\mathrm{d}_{2}\right)$ There exist two sequences $\left\{\xi_{k}\right\} \subseteq \mathbb{R}$ and $\left\{r_{k}\right\} \subseteq \mathbb{R}^{+}$with $\lim _{k \rightarrow+\infty} r_{k}=+\infty$ such that

$$
\begin{gathered}
F\left(\xi_{k}\right)=\inf _{|\xi| \leqslant c\left(p r_{k}\right)^{1 / p}} F(\xi) \quad \forall k \in \mathbb{N}, \\
\frac{1}{p}\|a\|_{L^{1}(\Omega)}\left|\xi_{k}\right|^{p}+\|\beta\|_{L^{1}(\Omega)} G\left(\xi_{k}\right)<r_{k} \quad \forall k \in \mathbb{N} .
\end{gathered}
$$

$\left(d_{3}\right)$

$$
\liminf _{|\xi| \rightarrow+\infty} \frac{\|\alpha\|_{L^{1}(\Omega)} F(\xi)+\|\beta\|_{L^{1}(\Omega)} G(\xi)}{|\xi|^{p}}<-\frac{1}{p}\|a\|_{L^{1}(\Omega)} .
$$

In addition suppose that the following hold.

$\left(\mathrm{d}_{4}\right)$ The functions $l^{-}(x, t)$ and $l^{+}(x, t)$ are superpositionally measurable.

$\left(\mathrm{d}_{5}\right)$ For almost every $x \in \Omega$ and every $t \in D_{f} \cup D_{g}$,

$$
l^{-}(x, t) \leqslant 0 \leqslant l^{+}(x, t) \Longrightarrow l(x, t)=0,
$$

where

$$
l(x, t):=a(x)|t|^{p-2} t+\alpha(x) f(t)+\beta(x) g(t) \quad \forall(x, t) \in \Omega \times \mathbb{R} .
$$

Then problem $\left(N_{p}\right)$ admits an unbounded sequence of weak solutions. 
Proof. We begin by taking $X=W^{1, p}(\Omega), \tilde{X}=C^{\circ}(\bar{\Omega})$. Since $p>n, X$ is compactly embedded in $C^{\circ}(\bar{\Omega})$. Set

$$
\begin{aligned}
\Phi(u) & :=\int_{\Omega} \alpha(x) F(u(x)) \mathrm{d} x, \\
\Psi(u) & :=\frac{1}{p}\|u\|^{p}+\int_{\Omega} \beta(x) G(u(x)) \mathrm{d} x
\end{aligned}
$$

and

$$
J(u):=\Phi(u)+\Psi(u)
$$

for each $u \in X$. Clearly, $f, g \in L_{\text {loc }}^{\infty}(\mathbb{R})$ ensures that the above functionals are locally Lipschitz on $X$. So, it makes sense to consider their generalized directional derivatives $\Phi^{\circ}$, $\Psi^{\circ}$ and $J^{\circ}$, respectively. We claim that the functional $J$ admits an unbounded sequence $\left\{u_{k}\right\} \subseteq X$ of critical points (local minima). To this end, we apply Theorem 2.3 (b). It is a simple matter to verify that $\Psi$ is weak sequentially lower semicontinuous on $X$. Bearing in mind that $\beta(x) \geqslant 0$ a.e. in $\Omega$, by $\left(\mathrm{d}_{1}\right)$ one has

$$
\Psi(u) \geqslant \frac{1}{p}\|u\|^{p} \quad \forall u \in X .
$$

Hence, $\Psi$ is coercive and $\inf _{X} \Psi=\Psi(0)=0$.

Now, using the notation of Theorem 2.3 and arguing as in the proof of Theorem 2.1 of [10] we obtain that $\gamma=0$. For this purpose, taking into account that $\lim _{k \rightarrow+\infty} r_{k}=+\infty$ and $\varphi(\rho) \geqslant 0 \forall \rho>0$, it is enough to show that

$$
\varphi\left(r_{k}\right)=0 \quad \forall k \in \mathbb{N} .
$$

Clearly, through (3.3) one has that

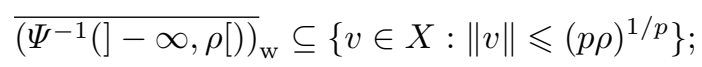

consequently,

$$
0 \leqslant \varphi(\rho) \leqslant \inf _{u \in \Psi^{-1}(]-\infty, \rho[)} \frac{\Phi(u)-\inf _{\|v\| \leqslant(p \rho)^{1 / p}} \Phi(v)}{\rho-\Psi(u)} \quad \forall \rho>0 .
$$

As the embedding constant $c$ is finite, $\|v\| \leqslant\left(p r_{k}\right)^{1 / p}$ produces

$$
|v(x)| \leqslant c\left(p r_{k}\right)^{1 / p} \quad \text { in } \Omega .
$$

Equation (3.1) then implies that $F(v(x)) \geqslant F\left(\xi_{k}\right)$ for each $x \in \Omega$. Moreover, owing to (3.2) one has $\xi_{k} \in \Psi^{-1}(]-\infty, r_{k}[)$. Thus,

$$
\Phi\left(\xi_{k}\right)=\inf _{\|v\| \leqslant\left(p r_{k}\right)^{1 / p}} \Phi(v)
$$

$(\alpha(x) \geqslant 0$ a.e. in $\Omega)$. Therefore, (3.4) holds. 
In applying Theorem 2.3 the last step is to verify that $J$ is unbounded below. To do this take

$$
\eta \in] \frac{1}{p}\|a\|_{L^{1}(\Omega)},-\liminf _{|\xi| \rightarrow+\infty} \frac{\|\alpha\|_{L^{1}(\Omega)} F(\xi)+\|\beta\|_{L^{1}(\Omega)} G(\xi)}{|\xi|^{p}}[
$$

and choose a sequence $\left\{\sigma_{k}\right\} \subseteq \mathbb{R}$ such that

$$
\lim _{k \rightarrow+\infty}\left|\sigma_{k}\right|=+\infty, \quad\|\alpha\|_{L^{1}(\Omega)} F\left(\sigma_{k}\right)+\|\beta\|_{L^{1}(\Omega)} G\left(\sigma_{k}\right)<-\eta\left|\sigma_{k}\right|^{p} \quad \forall k \in \mathbb{N} .
$$

Bearing in mind that $\left\|\sigma_{k}\right\|^{p}=\|a\|_{L^{1}(\Omega)}\left|\sigma_{k}\right|^{p}$, an easy computation shows that

$$
J\left(\sigma_{k}\right)<\left(\frac{1}{p}\|a\|_{L^{1}(\Omega)}-\eta\right)\left|\sigma_{k}\right|^{p} .
$$

Therefore, $\lim _{k \rightarrow+\infty} J\left(\sigma_{k}\right)=-\infty$. Choosing $\lambda=1$, Theorem 2.3 gives a sequence $\left\{u_{k}\right\} \subseteq$ $X$ such that $\lim _{k \rightarrow+\infty} \Psi\left(u_{k}\right)=+\infty$, as well as, for each point $u_{k}$,

$$
J^{\circ}\left(u_{k} ; v\right) \geqslant 0 \quad \forall v \in X .
$$

Furthermore, $\Psi$ is bounded on bounded sets, so $\left\{u_{k}\right\}$ is unbounded. At this point, pick $k \in \mathbb{N}$ and take into account that the functional

$$
h(u):=\frac{1}{p}\|u\|^{p} \quad \forall u \in X
$$

is continuously Gâteaux differentiable with

$$
h^{\prime}(u)(v)=\int_{\Omega}\left(\left|\nabla u_{k}(x)\right|^{p-2} \nabla u_{k}(x) \nabla v(x)+a(x)\left|u_{k}(x)\right|^{p-2} u_{k}(x) v(x)\right) \mathrm{d} x .
$$

Then the preceding inequality (3.5) implies

$$
-\int_{\Omega}\left(\left|\nabla u_{k}(x)\right|^{p-2} \nabla u_{k}(x) \nabla v(x)\right) \mathrm{d} x \leqslant \int_{\Omega}\left(a(x)\left|u_{k}(x)\right|^{p-2} u_{k}(x) v(x)\right) \mathrm{d} x+T^{\circ}\left(u_{k}, v\right),
$$

for each $v \in X$, where $T$ is the locally Lipschitz functional defined in $X$ by putting

$$
T(u):=\int_{\Omega}\left(\int_{0}^{u(x)} \alpha(x) f(t)+\beta(x) g(t) \mathrm{d} t\right) \mathrm{d} x \quad \forall u \in X .
$$

On the other hand, the integral

$$
\int_{\Omega}\left(a(x)\left|u_{k}(x)\right|^{p-2} u_{k}(x) v(x)\right) \mathrm{d} x
$$

represents the derivative at $u_{k}$ of the continuously Gâteaux differentiable functional

$$
h_{1}(u):=\int_{\Omega} \mathrm{d} x \int_{0}^{u(x)} a(x)|t|^{p-2} t \mathrm{~d} t
$$


for each $u \in X$. Thus, owing to Proposition 2.1, the right-hand side of (3.7) becomes the generalized derivative of the following locally Lipschitz functional $H$, defined in $X$ by setting

$$
H(u):=\int_{\Omega}\left(\int_{0}^{u(x)} l(x, t) \mathrm{d} t\right) \mathrm{d} x \quad \forall u \in X .
$$

Hence,

$$
-\int_{\Omega}\left(\left|\nabla u_{k}(x)\right|^{p-2} \nabla u_{k}(x) \nabla v(x)\right) \mathrm{d} x \leqslant H^{\circ}\left(u_{k}, v\right) \quad \forall v \in X,
$$

which actually means

$$
-\Delta_{p} u_{k} \in \partial H\left(u_{k}\right)
$$

Moreover, through standard arguments on regularity (see for instance Theorem 6.1 of [8]) one has

$$
-\Delta_{p} u_{k} \in W^{1, p^{\prime}}(\Omega),
$$

while Theorem 2.1 of $[4]$ yields

$$
-\Delta_{p} u_{k}(x) \in\left[l^{-}\left(x, u_{k}(x)\right), l^{+}\left(x, u_{k}(x)\right)\right] \quad \text { a.e. in } \Omega .
$$

Finally, by continuity we have

$$
-\Delta_{p} u_{k}(x)=l\left(x, u_{k}(x)\right)
$$

whenever $x \in \Omega \backslash u_{k}^{-1}\left(D_{f} \cup D_{g}\right)$. Whereas Proposition 2.2 produces

$$
-\Delta_{p} u_{k}(x)=0 \quad \text { a.e. in } u_{k}^{-1}\left(D_{f} \cup D_{g}\right) .
$$

So, according to $\left(d_{5}\right)$ one has

$$
-\Delta_{p} u_{k}(x)=0=l\left(x, u_{k}(x)\right) \quad \text { a.e. in } u_{k}^{-1}\left(D_{f} \cup D_{g}\right) .
$$

In any case we achieve

$$
-\Delta_{p} u_{k}(x)=l\left(x, u_{k}(x)\right) \quad \text { a.e. in } \Omega .
$$

This completes the proof.

Now, arguing in the same way as in Theorem 3.1, by Theorem 2.3 (c) and replacing both $r_{k} \rightarrow+\infty$ with $r_{k} \rightarrow 0^{+}$and $|\xi| \rightarrow+\infty$ with $|\xi| \rightarrow 0^{+}$it is possible to prove the following theorem.

Theorem 3.2. Let $f, g: \mathbb{R} \rightarrow \mathbb{R}$ be two locally essentially bounded functions, which are possibly discontinuous in a set having Lebesgue measure zero, namely $D_{f}$ and $D_{g}$, respectively. Assume that the following assertions hold.

$\left(\mathrm{d}_{1}^{*}\right) \inf _{\xi \in \mathbb{R}} G(\xi) \geqslant 0$. 
$\left(\mathrm{d}_{2}^{*}\right)$ There exist two sequences $\left\{\xi_{k}\right\} \subseteq \mathbb{R}$ and $\left\{r_{k}\right\} \subseteq \mathbb{R}^{+}$with $\lim _{k \rightarrow+\infty} r_{k}=0^{+}$such that

$$
\begin{gathered}
F\left(\xi_{k}\right)=\inf _{|\xi| \leqslant c\left(p r_{k}\right)^{1 / p}} F(\xi) \quad \forall k \in \mathbb{N}, \\
\frac{1}{p}\|a\|_{L^{1}(\Omega)}\left|\xi_{k}\right|^{p}+\|\beta\|_{L^{1}(\Omega)} G\left(\xi_{k}\right)<r_{k} \quad \forall k \in \mathbb{N} .
\end{gathered}
$$

$\left(d_{3}^{*}\right)$

$$
\liminf _{|\xi| \rightarrow 0^{+}} \frac{\|\alpha\|_{L^{1}(\Omega)} F(\xi)+\|\beta\|_{L^{1}(\Omega)} G(\xi)}{|\xi|^{p}}<-\frac{1}{p}\|a\|_{L^{1}(\Omega)} .
$$

In addition suppose that the following assertions hold.

$\left(\mathrm{d}_{4}^{*}\right)$ The functions $l^{-}(x, t)$ and $l^{+}(x, t)$ are superpositionally measurable.

$\left(\mathrm{d}_{5}^{*}\right)$ For almost every $x \in \Omega$ and every $t \in D_{f} \cup D_{g}$,

$$
l^{-}(x, t) \leqslant 0 \leqslant l^{+}(x, t) \Longrightarrow l(x, t)=0,
$$

where

$$
l(x, t):=a(x)|t|^{p-2} t+\alpha(x) f(t)+\beta(x) g(t) \quad \forall(x, t) \in \Omega \times \mathbb{R} .
$$

Then problem $\left(N_{p}\right)$ admits a sequence of non-zero weak solutions which strongly converges to 0 .

Remark 3.3. We now explicitly observe that Theorems 3.1 and 3.2 extend the main results of $[\mathbf{1 3}]$.

\section{Examples}

Here, we give three examples which show how the results presented in the preceding section are successfully applied in solving problem $\left(N_{p}\right)$ in several concrete cases.

Example 4.1. Let $C$ be a closed subset of $] 0, \pi / 2[$ such that $m(C)=0$. Then the set $] 0, \pi / 2$ [ is non-empty and open. So, it has at most countably many connected (open) components, namely \{]$a_{k}, b_{k}[\}_{k \in \mathbb{N}}$. Without loss of generality we can assume that $b_{k}<$ $a_{k+1}$ for each $k \in \mathbb{N}$. Define, for every $t \in \mathbb{R}$,

$$
f(t):= \begin{cases}\mathrm{e}^{t}(\sin t+\cos t-1) & \text { if } t \in[\pi / 2,+\infty[ \\ y_{k} & \text { if } t \in] a_{k}, b_{k}[ \\ 0 & \text { otherwise }\end{cases}
$$

where $\left\{y_{k}\right\}_{k \in \mathbb{N}}$ is a bounded sequence complying with $\inf _{k \in \mathbb{N}} y_{k}>0$, and $g(t):=0$. Moreover, suppose that

$$
\|a\|_{L^{1}(\Omega)}=1 / c^{p} .
$$

Then the conclusion of Theorem 3.1 holds. 
Proof. Obviously, one has $G(\xi)=0$ for each $\xi \in \mathbb{R}$ and a straightforward computation yields

$$
F(\xi):= \begin{cases}0 & \text { if } \left.\xi \in]-\infty, a_{0}\right], \\ \sum_{j=0}^{k-1} y_{j}\left(b_{j}-a_{j}\right)+\left(\xi-a_{k}\right) y_{k} & \text { if } \xi \in] a_{k}, b_{k}[, \\ \mathrm{e}^{\xi}(\sin \xi-1)+s & \text { if } \xi \in[\pi / 2,+\infty[\end{cases}
$$

where

$$
s=\sum_{k=0}^{+\infty} y_{k}\left(b_{k}-a_{k}\right)<+\infty .
$$

Now, setting, for each $k \in \mathbb{N}$,

$$
\eta_{k}:=\frac{3}{2} \pi+2 k \pi, \quad \mu_{k}:=\frac{5}{2} \pi+2 k \pi, \quad r_{k}:=\frac{1}{p}\left(\frac{\mu_{k}}{c}\right)^{p} .
$$

we obtain, for some $\left.\xi_{k} \in\right]-\eta_{k}, \eta_{k}[$,

$$
\inf _{|\xi| \leqslant c\left(\eta_{k}\right)^{1 / p}} F(\xi)=\inf _{|\xi| \leqslant c\left(p r_{k}\right)^{1 / p}} F(\xi)=F\left(\xi_{k}\right),
$$

together with $\lim _{k \rightarrow+\infty} r_{k}=+\infty$. So, by using (4.1), condition (3.2) can easily be drawn. Thus, $\left(\mathrm{d}_{2}\right)$ holds. Moreover, we achieve

$$
\liminf _{|\xi| \rightarrow+\infty} \frac{\|\alpha\|_{L^{1}(\Omega)} F(\xi)+\|\beta\|_{L^{1}(\Omega)} G(\xi)}{|\xi|^{p}} \leqslant-\|\alpha\|_{L^{1}(\Omega)} \lim _{k \rightarrow+\infty} \frac{2 \mathrm{e}^{\eta_{k}}-s}{\eta_{k}^{p}}=-\infty,
$$

which implies $\left(d_{3}\right)$, while $\left(d_{4}\right)$ follows from elementary arguments on measurability. Let us note that

$$
D_{f}=C .
$$

Then, since $f(t) \geqslant 0$, provided $t \in] 0, \pi / 2[$ and $f(0)=0$, we obtain $l(x, t) \geqslant \theta>0$ a.e. in $(x, t) \in \Omega \times] 0, \pi / 2[$ and $l(x, 0)=0$ for each $x \in \Omega$. This actually means

$$
l(x, 0)=0<l^{-}\left(x, a_{k}\right)<l^{-}\left(x, b_{k}\right),
$$

for each $k \in \mathbb{N}$ and a.e. in $\Omega$. In any case condition $\left(\mathrm{d}_{5}\right)$ is fulfilled. This completes the proof.

Example 4.2. Let $f, g: \mathbb{R} \rightarrow \mathbb{R}$ be defined by setting

$$
f(t):= \begin{cases}\frac{t}{|t|}\left(\mathrm{e}^{|t|}-1\right)^{p-1}\left(\mathrm{e}^{|t|} \sin \frac{1}{|t|^{p-1}}-\frac{(p-1)}{p} \frac{\left(\mathrm{e}^{|t|}-1\right)}{|t|^{p}} \cos \frac{1}{|t|^{p-1}}\right) & \text { if } t \neq 0, \\ 0 & \text { otherwise }\end{cases}
$$

and $g(t):=0$ for every $t \in \mathbb{R}$. Assume that

$$
\|a\|_{L^{1}(\Omega)}=\frac{1}{c^{p}}<\|\alpha\|_{L^{1}(\Omega)} .
$$

Then the conclusion of Theorem 3.2 holds. 
Proof. Clearly, $\left(\mathrm{d}_{1}^{*}\right)$ and $\left(\mathrm{d}_{4}^{*}\right)$ are trivial. Furthermore, we have

$$
F(t):= \begin{cases}\frac{1}{p}\left(\mathrm{e}^{|t|}-1\right)^{p} \sin \frac{1}{|t|^{p-1}} & \text { if } t \neq 0 \\ 0 & \text { otherwise. }\end{cases}
$$

Thus, writing

$$
a_{k}=\left(\frac{1}{(2 k+1) \pi}\right)^{1 / p-1}, \quad b_{k}=\left(\frac{1}{2 k \pi}\right)^{1 / p-1}, \quad k \in \mathbb{N}^{*},
$$

results in

$$
\inf _{|\xi| \leqslant b_{k}} F(\xi)=\inf _{|\xi| \leqslant a_{k}} F(\xi)=F\left(\xi_{k}\right)
$$

for some $\xi_{k} \in\left[-a_{k}, a_{k}\right]$. Now, set

$$
\xi_{0}:=0, \quad r_{0}:=1, \quad r_{k}:=\frac{1}{p}\left(\frac{b_{k}}{c}\right)^{p}, \quad k \geqslant 1,
$$

and observe that $r_{k} \rightarrow 0^{+}$as $k \rightarrow+\infty$. According to the above results and bearing in mind (4.2) we can assert that $\left(\mathrm{d}_{2}^{*}\right)$ holds. Moreover, write, for each $k \in \mathbb{N}$,

$$
\mu_{k}=\left(\frac{2}{(4 k+3) \pi}\right)^{1 /(p-1)}
$$

It is easy to verify that

$$
\liminf _{|\xi| \rightarrow 0^{+}} \frac{F(\xi)}{|\xi|^{p}} \leqslant \lim _{k \rightarrow+\infty} \frac{F\left(\mu_{k}\right)}{\mu_{k}^{p}}=-\frac{1}{p}
$$

from which we obtain $\left(\mathrm{d}_{3}^{*}\right)$. Let us note that $D_{f}=\{0\}$. Next, a trivial verification show that

$$
l^{-}(x, 0)=-\frac{p-1}{p} \alpha(x), \quad l^{+}(x, 0)=\frac{p-1}{p} \alpha(x) \quad \text { a.e. in } \Omega .
$$

So $l(x, 0)=0$ implies $\left(\mathrm{d}_{5}^{*}\right)$. Since all the assumptions of Theorem 3.2 are satisfied, the conclusion follows.

Example 4.3. Let $\Omega$ be a convex set, whose diameter is less than or equal to

$$
n^{1 / p}\left(\frac{p-n}{p-1}\right)^{1-(1 / p)}
$$

Let $\left\{a_{k}\right\}_{k \in \mathbb{N}}$ be a real sequence such that $a_{k+1}=4 a_{k}$ for each $k \in \mathbb{N}$, with $a_{0}=2$. Put $a(x)=\alpha(x)=1$ for almost every $x$ in $\Omega$ and define, for every $t \in \mathbb{R}$,

$$
f(t):= \begin{cases}a_{k}^{p-2}\left(1-2^{p-1}\right)\left(t-a_{k}\right)-a_{k}^{p-1} & \text { if } t \in\left[a_{k}, 2 a_{k}\right], \\ 0 & \text { otherwise, }\end{cases}
$$

and $g(t):=0$. Then the conclusion of Theorem 3.1 holds. 
Proof. It is now evident that $\left(\mathrm{d}_{1}\right)$ and $\left(\mathrm{d}_{4}\right)$ are true. An easy verification show that

$$
\int_{a_{k}}^{a_{k+1}} f(t) \mathrm{d} t=-\frac{2^{p-1}+1}{2} a_{k}^{p}
$$

Indeed, we obtain

$$
F\left(a_{k+1}\right)=F\left(2 a_{k}\right)=-\sum_{j=0}^{k-1} \frac{2^{p-1}+1}{2} a_{j}^{p} .
$$

Now, let us define, provided that $k \in \mathbb{N}$,

$$
\xi_{k}:=2 a_{k}, \quad r_{k}:=\frac{1}{p}\left(\frac{a_{k+1}}{c}\right)^{p} .
$$

Clearly, we have $r_{k} \rightarrow+\infty$ as $k \rightarrow+\infty$ and, taking into account that $F$ is non-increasing, one has

$$
F\left(\xi_{k}\right)=\inf _{|\xi| \leqslant c\left(p r_{k}\right)^{1 / p}} F(\xi) .
$$

In addition, (2.3) produces $m(\Omega) c^{p}<2^{p-1}$. From this, (3.2) follows. So $\left(\mathrm{d}_{2}\right)$ holds. Indeed, it results in

$$
F\left(\xi_{k}\right)<-\frac{2^{p-1}+1}{2} a_{k}^{p} \quad \forall k \in \mathbb{N}
$$

Thus, we achieve

$$
\liminf _{|\xi| \rightarrow+\infty} \frac{F(\xi)}{|\xi|^{p}} \leqslant \lim _{k \rightarrow+\infty} \frac{F\left(\xi_{k}\right)}{\xi_{k}^{p}}=-\frac{2^{p-1}+1}{2^{p+1}}<-\frac{1}{p},
$$

which implies $\left(d_{3}\right)$. Finally, we verify $\left(d_{5}\right)$. Let us note that

$$
D_{f}=\cup_{k \in \mathbb{N}}\left\{a_{k}, 2 a_{k}\right\} .
$$

Furthermore, a straightforward computation yields

$$
0=l^{-}\left(x, a_{k}\right)=l^{-}\left(x, 2 a_{k}\right)=l\left(x, a_{k}\right)=l\left(x, 2 a_{k}\right)
$$

for each $k \in \mathbb{N}$ and a.e. $x \in \Omega$. Since all the assumptions of Theorem 3.1 are satisfied, the conclusion follows.

Acknowledgements. The author thanks Professor S. A. Marano for introducing him to the topics treated in this paper and for many stimulating conversations.

\section{References}

1. P. A. Binding, P. Drábek And Y. X. HuAng, Existence of multiple solutions of critical quasilinear elliptic Neumann problems, Nonlin. Analysis 42 (2000), 613-629.

2. S. M. Bouguima, A quasilinear elliptic problem with a discontinuous nonlinearity, Nonlin. Analysis 11 (1995), 1115-1121. 
3. V. I. Burenkov And V. A. Gusakov, On precise constants in embedding theorems, Sov. Math. Dokl. 35 (1987), 651-655.

4. K. C. Chang, Variational methods for non-differentiable functionals and their applications to partial differential equations, J. Math. Analysis Applic. 80 (1981), 102-129.

5. F. H. Clarke, Optimization and nonsmooth analysis, Classics in Applied Mathematics, vol. 5 (SIAM, Philadelphia, PA, 1990).

6. E. De Giorgi, G. Buttazzo and C. Dal Maso, On the lower semicontinuity of certain integral functionals, Atti Accad. Naz. Lincei 74 (1983), 274-282.

7. N. C. Kourigenis AND N. S. PAPAgEORgiou, Three non trivial solutions for a quasilinear elliptic differential equation at resonance with discontinuous right hand side, J. Math. Analysis Applic. 238 (1999), 477-490.

8. J. L. Lions And E. Magenes, Problemi ai limiti non omogenei, Annli Scuola Norm. Sup. Pisa 16 (1962), 1-44.

9. S. A. Marano And D. Motreanu, On a three critical points theorem for nondifferentiable functions and applications to non linear boundary value problems, Nonlin. Analysis 48 (2002), 37-52.

10. S. A. Marano And D. Motreanu, Infinitely many critical points of non-differentiable functions and applications to a Neumann type problem involving the $p$-Laplacian, J. Diff. Eqns, in press.

11. D. Motreanu and P. D. Panagiotopoulos, Minimax theorems and qualitative properties of the solutions of hemivariational inequalities, Nonconvex Optimization Applications, vol. 29 (Kluwer, Dordrecht, 1998).

12. B. RiCCERI, A general variational principle and some of its applications, J. Comput. Appl. Math. 113 (2000), 401-410.

13. B. RICCERI, Infinitely many solutions of the Neumann problem for elliptic equations involving the p-Laplacian, Bull. Lond. Math. Soc. 33 (2001), 331-340. 